九州大学学術情報リポジトリ

Kyushu University Institutional Repository

\title{
Mathematical Structure Common to Animal Growth Analysis and Space Expansion Analysis
}

Shimojo, Masataka

Laboratory of Regulation in Metabolism and Behavior, Division of Animal and Marine Bioresource

Sciences, Department of Bioresource Sciences, Faculty of Agriculture, Kyushu University

https://doi.org/10.5109/1564087

出版情報: 九州大学大学院農学研究院紀要. 61 (1)，pp.89-93，2016-02-29. Faculty of Agriculture， Kyushu University

バージョン :

権利関係 : 


\title{
Mathematical Structure Common to Animal Growth Analysis and Space Expansion Analysis
}

\author{
Masataka SHIMOJO* \\ Laboratory of Regulation in Metabolism and Behavior, Division of Animal and Marine Bioresource Sciences, \\ Department of Bioresource Sciences, Faculty of Agriculture, Kyushu University, \\ Fukuoka 812-8581, Japan \\ (Received September 28, 2015 and accepted November 19, 2015)
}

\begin{abstract}
This study was conducted to investigate mathematical structures common to animal growth analysis and space expansion analysis. Six particular functions for the growth analysis of the individual animal had a common mathematical structure composed of the respective function and its first and second derivatives. The standard model of the space expansion had a deceleration parameter composed of the scale factor and its first and second derivatives. The results obtained from this study suggested hypotheses $(\alpha) \sim(\gamma)$. $(\alpha)$ The mathematical structure common to six functions for the animal growth analysis was the same as that of the deceleration parameter for the cosmic expansion analysis, except for the reciprocal form with the negative sign in the latter case. This similarity was based on the symmetry that exponential function showed about the differential calculation at the back of both analyses. ( $\beta$ ) There were inflection points both in five functions for the animal growth analysis and in the cosmic expansion history. $(\gamma)$ If the present size of the space was normalized as ' 1 ', then there was a dual interpretation that the cosmic expansion under the general relativity was related to the object recession under the special relativity.
\end{abstract}

Key words: animal growth, common structure, dual interpretation, inflection point, space expansion

\section{INTRODUCTION}

Many kinds of functions have so far been reported for the growth analysis of the individual animal or plant. Among them, several particular functions composed of exponential function are often used, and their common root is in Bertalanffy function based on the anabolism and catabolism (Bertalanffy, 1949, 1957; Richards, 1959; Osumi and Ishikawa,1983). This was reconfirmed by a subsequent study (Shimojo et al., 2012) that suggested a mathematical structure common to those particular functions.

By the way, there is an interesting study (Tegmark, 2014) that our baby universe based on the inflation theory grew much like a human baby. Exponential function describes the inflationary universe (Starobinsky, 1980; Kazanas, 1980; Sato, 1981; Guth, 1981; Linde, 1982; Albrecht and Steinhardt, 1982), and may also describe the reaccelerating universe that began several billion years ago (Riess et al., 1998; Perlmutter et al., 1999). Shimojo (2014, 2015a, 2015b) interpreted Bondi K-factor (Bondi, 1964) as a toy model for the space expansion study, using a relationship between Bondi K-factor and exponential function.

The present study was designed to investigate the mathematical structure common to animal growth analysis and space expansion analysis, by adding a few pieces of information to previous studies (Shimojo et al., 2012; Shimojo, 2014, 2015a, 2015b).

*E-mail: mshimojo@agr.kyushu-u.ac.jp

This study was conducted mostly at Agri-Bio Research Laboratory on Ito Campus, Kyushu University, Motooka, Fukuoka 819-0395

\section{ANIMAL GROWTH ANALYSIS}

\section{Six particular functions}

Shimojo et al. (2012) investigated six particular functions (1) (6) by citing some preceding studies (Brody, 1945; Bertalanffy, 1949, 1957; Richards, 1959; Osumi and Ishikawa, 1983); Bertalanffy $\left(W_{\mathrm{v}}\right)$, Richards $\left(W_{\mathbf{R}}\right)$, Mitscherlich $\left(W_{\mathbf{M}}\right)$, logistic $\left(W_{\mathbf{L}}\right)$, Gompertz $\left(W_{\mathbf{G}}\right)$ and basic growth $\left(W_{\mathbf{B}}\right)$ functions,

$$
\begin{aligned}
& W_{\mathbf{V}}=\left(\alpha / \beta-\left(\alpha / \beta-W_{\mathbf{0}}^{1-m}\right) \exp (-\beta(1-m) t)\right)^{1 /(1-m)} \\
& W_{\mathbf{R}}=A(1-b \exp (-k t))^{1 /(1-m)} \\
& W_{\mathbf{M}}=A(1-b \exp (-k t)) \\
& W_{\mathbf{L}}=\frac{A}{1+b \exp (-k t)} \\
& W_{\mathbf{G}}=A \exp (-b \exp (-k t)) \\
& W_{\mathbf{B}}=W_{\mathbf{0}} \exp (r t)
\end{aligned}
$$

where $\alpha=$ anabolic constant, $\beta=$ catabolic constant, $W=$ weight, $t=$ time, $A, b, k$ and $m$ are constants, $W_{0}^{1-m}=$ weight at $t=0, W_{\mathbf{0}}=$ weight at $t=0, r=$ relative growth rate.

A mathematical structure common to six functions Shimojo et al. (2012) suggested a mathematical structure (7) common to six functions,

$$
\frac{(d W / d t)^{2}}{W\left(d^{2} W / d t^{2}\right)} \text {. }
$$


Thus, applying (7) to six functions gives,

$$
\begin{aligned}
& \frac{\left(d W_{\mathbf{v}} / d t\right)^{2}}{W_{\mathbf{v}}\left(d^{2} W_{\mathbf{v}} / d t^{2}\right)} \\
& =\frac{\left(\alpha / \beta-W_{0}^{1-m}\right) \exp (-\beta(1-m) t)}{\left(\alpha / \beta-W_{0}^{1-m}\right) \exp (-\beta(1-m) t)-(\alpha / \beta)(1-m)}, \\
& \frac{\left(d W_{\mathbf{R}} / d t\right)^{2}}{W_{\mathbf{R}}\left(d^{2} W_{\mathbf{R}} / d t^{2}\right)}=\frac{b \exp (-k t)}{b \exp (-k t)-(1-m)}, \\
& \frac{\left(d W_{\mathbf{M}} / d t\right)^{2}}{W_{\mathbf{M}}\left(d^{2} W_{\mathbf{M}} / d t^{2}\right)}=\frac{b \exp (-k t)}{b \exp (-k t)-1}, \\
& \frac{\left(d W_{\mathbf{L}} / d t\right)^{2}}{W_{\mathbf{L}}\left(d^{2} W_{\mathbf{L}} / d t^{2}\right)}=\frac{b \exp (-k t)}{b \exp (-k t)-1}, \\
& \frac{\left(d W_{\mathbf{G}} / d t\right)^{2}}{W_{\mathbf{G}}\left(d^{2} W_{\mathbf{G}} / d t^{2}\right)}=\frac{b \exp (-k t)}{b \exp (-k t)-1}, \\
& \frac{\left(d W_{\mathbf{B}} / d t\right)^{2}}{W_{\mathbf{B}}\left(d^{2} W_{\mathbf{B}} / d t^{2}\right)}=1 \text {. }
\end{aligned}
$$

The mathematical structure common to the left-hand side of six expressions (1-1) (6-1) gives mathematical features (i) (iv) to the right-hand side of them. (i) The mathematical structure (7) gives a unified viewpoint, from which six functions are classified into theree categories by operating parameters; category-1 (Bertalanffy, Richards), category-2 (Mitscherlich, logistic, Gompertz), and category-3 (basic growth). Functions (1) (5) in categories 1 and 2 have inflection points, but function (6) does not have. (ii) Expression (6-1) gives '1', a symmetry that basic growth function (6) without an inflection point shows about the differential calculation. Expressions (1-1) (5-1) give complexities of five functions $(1) \sim(5)$, as differences from the symmetry (6-1) that basic growth function (6) shows. Those differences regulate the exponential increase to give the aymptotic growth that the individual animal actually shows. (iv) Expressions (3-1) (5-1) give a common structure hidden at the back of three functions (3) (5) that are different in the position of the inflection point.

\section{SPACE EXPANSION ANALYSIS (I)}

\section{Deceleration parameter in the standard model of the cosmic expansion}

The standard model of the cosmic expansion is given by expressions (8) and (9),

$$
H^{2}=\left(\frac{1}{a} \cdot \frac{d a}{d t}\right)^{2}
$$

$$
\begin{gathered}
=\frac{8 \pi G}{3 c^{2}} \rho-\frac{c^{2} K}{a^{2}}+\frac{c^{2} \Lambda}{3}, \\
\frac{1}{a} \cdot \frac{d^{2} a}{d t^{2}}=-\frac{4 \pi G}{3 c^{2}}(\rho+3 p)+\frac{c^{2} \Lambda}{3},
\end{gathered}
$$

where $H=$ Hubble parameter (cosmic expansion rate), $a$ = cosmic scale factor, $t=$ time, $\pi=$ circular constant, $G=$ gravitational constant, $c=$ speed of light in vacuum, $\rho=$ energy density, $K=$ curvature of space, $\Lambda$ = cosmological constant, $p=$ pressure.

The deceleration parameter $(q)$ of this standard cosmic model is defined by,

$$
q=-\frac{a\left(d^{2} a / d t^{2}\right)}{(d a / d t)^{2}} .
$$

When the deceleration parameter takes negative values, the universe expands.

\section{ANIMAL GROWTH ANALYSIS AND SPACE EXPANSION ANALYSIS}

\section{Mathematical structures common to animal growth analysis and space expansion analysis}

The comparison will be made between expression (7) for the animal growth analysis and expression (10) for the space expansion analysis,

$$
\frac{(d W / d t)^{2}}{W\left(d^{2} W / d t^{2}\right)}, \text { (7) } q=-\frac{a\left(d^{2} a / d t^{2}\right)}{(d a / d t)^{2}}
$$

where $W=$ weight of the individual animal, $a=$ cosmic scale factor.

Expressions (7) and (10) suggest the same mathematical structure, except for the reciprocal form and the negative sign in the cosmic deceleration parameter $(q)$. This might suggest a mathematical hypothesis that the symmetry that exponential function shows about the differential calculation is hidden at the back of both analyses.

In addition, there are inflection points both in five particular functions for the animal growth analysis (Bertalanffy, 1949, 1957; Richards, 1959; Osumi and Ishikawa,1983) and in the cosmic expansion history (Riess et al., 1998; Perlmutter et al., 1999).

\section{SPACE EXPANSION ANALYSIS (II)}

\section{Relationship between cosmological redshift and relativistic Doppler redshift}

This issue is taken up again in this study, because there are problems in previous studies (Shimojo, 2014, 2015a, 2015b).

The redshift $(Z)$ is given by the relative difference between the observed wavelength $\left(\lambda_{\mathrm{o}}\right)$ and the emitted wavelength $\left(\lambda_{\mathbf{E}}\right)$ of an object, 


$$
Z=\frac{\lambda_{\mathbf{o}}}{\lambda_{\mathrm{E}}}-1
$$

The cosmological redshift $\left(Z_{\mathrm{c}}\right)$ is due to the increase of the cosmic scale factor $(a)$ in the whole period from the emission to the absorption of light (Weinberg, 2008) under the general relativity (FLRW metric),

$$
Z_{\mathbf{C}}=\frac{a\left(t_{\mathbf{o}}\right)}{a\left(t_{\mathbf{E}}\right)}-1
$$

However, the relativistic Doppler redshift $\left(Z_{\mathrm{R}}\right)$ is due to the light emitted from an object receding from an observer under the special relativity (Minkowski metric),

$$
Z_{\mathbf{R}}=\frac{1+v_{\mathbf{E}} / c}{\sqrt{1-\left(v_{\mathbf{E}} / c\right)^{2}}}-1=\sqrt{\frac{c+v_{\mathbf{E}}}{c-v_{\mathbf{E}}}}-1,
$$

where $v$ recessional velocity of an object, $c=$ speed of light in vacuum.

The term given by ' $Z_{\mathbf{R}}+1$ ' in (13) is often called Bondi K-factor (Bondi, 1964).

The redshift $(Z)$ is interpreted as the cosmological redshift $\left(Z_{\mathbf{c}}\right)$ under the general relativity or as the relativistic Doppler redshift $\left(Z_{\mathbf{R}}\right)$ under the special relativity. Thus, relating expressions (11) (13) gives expressions (14) and (15),

$$
\frac{\lambda_{\mathbf{o}}}{\lambda_{\mathbf{E}}}=\frac{a\left(t_{\mathbf{o}}\right)}{a\left(t_{\mathbf{E}}\right)},(14) \quad \frac{\lambda_{\mathbf{o}}}{\lambda_{\mathbf{E}}}=\sqrt{\frac{c+v_{\mathbf{E}}}{c-v_{\mathbf{E}}}} .
$$

Although the left-hand side is equal between (14) and (15), there is a conceptual difference between the righthand sides (namely $Z_{\mathbf{C}}$ versus $Z_{\mathrm{R}}$ ), as already reported by studies (for example; Bedran, 2001; Davis and Lineweaver, 2004; Lineweaver and Davis, 2005; Weinberg, 2008).

However, if $t_{\mathbf{o}}$ shows the present time, then $a\left(t_{\mathbf{o}}\right)=1$ (the relative size of the present universe) by normalization and expression (14) becomes (16),

$$
\frac{\lambda_{\mathbf{o}}}{\lambda_{\mathbf{E}}}=\frac{1}{a\left(t_{\mathbf{E}}\right)} .
$$

In addition, expression (15) is transformed into,

$$
\frac{\lambda_{\mathbf{o}}}{\lambda_{\mathbf{E}}}=\frac{1}{\sqrt{\frac{c-v_{\mathbf{E}}}{c+v_{\mathbf{E}}}}} .
$$

Comparing the right-hand side of (16) with that of (17) might suggest hypotheses (A) and (B). (A) The scale factor $a\left(t_{\mathbf{E}}\right)$ in (16) shows the relative size of the universe when the light is emitted. If the numerator of (17), ' 1 ', is interpreted as the relative size of the present space, then the denominator of (17) is related to the relative size of the space when the light is emitted. (B) Thus, there is a one-to-one correspondence between the value of $t$ under the general relativity and the value of $v$ under the special relativity, a dual interpretation (18) that the scale factor is related to the object recession,

$$
\frac{1}{a(t)}=\frac{1}{\sqrt{\frac{c-v}{c+v}}} .
$$

The space under the FLRW metric is dynamic but the space under the Minkowski metric is static, though both spaces are flat.

Shimojo (2014, 2015a, 2015b) suggested hypothetical interpretations; expression (19) as a toy model of the space, expression (20) as a toy model of the energy density, and expression (21) as a toy model of the invariant that controls the relationship between the space size and the energy density,

$$
\begin{aligned}
& \sqrt{\frac{c-v}{c+v}},(19) \quad \sqrt{\frac{c+v}{c-v}}=Z_{\mathbf{R}}+1=\frac{f_{\mathbf{E}}}{f_{\mathbf{O}}} . \\
& \sqrt{\frac{c-v}{c+v}} \cdot \sqrt{\frac{c+v}{c-v}}=\sqrt{\frac{c-v}{c+v}} \cdot\left(Z_{\mathbf{R}}+1\right) \\
&=\sqrt{\frac{c-v}{c+v}} \cdot \frac{f_{\mathbf{E}}}{f_{\mathbf{o}}}=1,
\end{aligned}
$$

where $c \geq v \geq 0, f=$ frequency of light.

Expressions (19) (21) might suggest a hypothetical space expansion (a) (f), using the dimensionless relative size and relative energy density. (a) The expansion history of the toy space model is as follows; singularity, early rapid expansion, decelerated expansion, inflection point, and accelerated expansion. (b) If the reduction of the energy density under the invariant is a natural principle, then the space is born and expands when $v$ begins to decrease from $c$. At the singularity $\left[v=c, Z_{\mathbf{R}}=\infty\right.$, size $=0$, energy density $\left.\left(Z_{\mathbf{R}}+1\right)=\infty, 0 \cdot \infty=1\right]$, the space is born from the quantum phenomenon through something like a renormalization of infinities $[(0 \cdot \infty=1) \rightarrow(-\infty+\infty$ $=2 n \pi \boldsymbol{i}), \boldsymbol{i}=$ imaginary unit]. (c) The energy in the space causes the space to expand. At the stage right after the birth of the space, there is a very high expansion rate of the space and this is accompanied by a very high reduction rate of the energy density. (d) The rapid and large reduction of the energy density is associated with the rapid and large formation of the dark matter and the baryonic matter. This gives the very strong attractive force, causing the decelerated expansion of the space. (e) However, the reduction rate of the energy density shows a slowdown with the decrease of $v$. This is associated with the slowdown of the formation rate of the dark matter and baryonic matter (namely, the attractive force). (f) There is an inflection point at the halfway point $[v=$ $c / 2, Z_{\mathbf{R}}=0.732$, size $=0.577$, energy density $\left(Z_{\mathbf{R}}+1\right)=$ 1.732] between the birth point $\left[\mathrm{v}=c, Z_{\mathbf{R}}=\infty\right.$, size $=0$, energy density $\left.\left(Z_{\mathbf{R}}+1\right)=\infty\right]$ and the present point $[v=$ $0, Z_{\mathbf{R}}=0$, size $=1$, energy density $\left.\left(Z_{\mathbf{R}}+1\right)=1\right]$. Thus, at this halfway point, the decelerated expansion by the attractive force (dark matter and baryonic matter) is 
changed into the accelerated expansion by the repulsive force (dark energy). This accelerated expansion is lasting even at the present. However, the energy density [= 1] at the present is much lower than the energy density $[\approx \infty]$ causing a very rapid expansion at the stage right after the birth of the space.

The above hypothetical phenomena (a) (f) obtained from the toy space model based on the special relativity might be similar to the observational phenomena reported already by some studies based on the general relativity (Perlmutter et al., 1999; Barrow, 2011; Perlmutter, 2011, 2015). This similarity might be due to the dual relationship between the scale factor and the toy space model.

Chodorowski (2006) investigated, using a relativistic Doppler shift, an empty universe as a particular Friedman model. There are preceding studies that may allow the use of the relativistic Doppler redshift, as well as the cosmological redshift (for example; Bunn and Hogg, 2009; Davis, 2010a, 2010b; May and Yu, 2013; Tsujikawa, 2013; Li, 2014; Pierseaux, 2014). The relativistic Doppler redshift might suggest how the flat space-time emerges from nothing under the decrease of $v$ from $c$ to 0 .

However, there are many problems with this toy space model; for example, the early rapid expansion under the decelerated expansion is not consistent with the inflationary expansion under the accelerated expansion, the energy distribution (baryonic matter, dark matter, dark energy) is not shown, the relationship between the recessional velocity and the space expansion remains to be investigated, the time dilation remains to be investigated.

\section{The moment when the space is born}

At the moment when the space is born, the standard cosmic model suggests an exponential increase (22),

$$
a(t) \propto \exp (H \cdot t)
$$

There is a hypothetical relationship between the scale factor and the toy space model (18),

$$
\frac{1}{a(t)}=\frac{1}{\sqrt{\frac{c-v}{c+v}}} .
$$

In addition, relating hyperbolic function to Lorentz factor gives a relationship between the toy space model and exponential function (23),

$$
\sqrt{\frac{c-v}{c+v}}=\exp (-\theta) .
$$

There is a one-to-one correspondence between the value of $v$ and the value of $\theta$ in (23).

Thus, relating expressions (18), (22) and (23) might suggest expression (24) that hypothetically relates the toy space model to the cosmic standard model,

$$
\sqrt{\frac{c-v}{c+v}} \propto \exp (-H \cdot t) .
$$

There is a one-to-one correspondence between $v$ and $H \cdot t$ in (24). If $v=c$, then $H=\infty$ under the condition that $t$ is finite. This might suggest that the cosmic expansion rate $(H)$ is much grater than the speed of light $(c)$ at the moment when the space is born. However, this phenomenon under the special relativity is not consistent with the general relativity that gives the superluminal rate of the cosmic expansion when the cosmological redshift is greater than 1.46 (Davis and Lineweaver, 2004).

\section{Hypothetical interpretations of expression (21)}

The natural logarithm of expression (21) gives expressions $(25) \sim(28)$,

$$
\begin{aligned}
& \sqrt{\frac{c-v}{c+v}} \cdot \sqrt{\frac{c+v}{c-v}}=1, \\
& \ln \sqrt{\frac{c-v}{c+v}}+\ln \sqrt{\frac{c+v}{c-v}} \\
& =2 n \pi \boldsymbol{i}, \\
& =2 n \pi \cdot \frac{\psi(x, t)-\psi^{*}(x, t)}{2 A \sin (x, t)}, \\
& =2 n \pi \cdot \frac{x p-p x}{h / 2 \pi}, \\
& =2 n \pi \cdot \lim _{\boldsymbol{v} \rightarrow \infty} \sqrt{\frac{c \pm v}{c \mp}},
\end{aligned}
$$

where $c \geq v \geq 0$ in (21) and (25) (27), $\boldsymbol{i}=$ imaginary unit, $x=$ position, $p=$ momentum, $\psi(x, t)=$ wave function, $\psi^{*}(x, t)=$ conjugate complex of $\psi(x, t), A=$ amplitude, $h=$ Planck constant

In expressions (25) (27), the left-hand side is 0 , but the right-hand sides might suggest quantum phenomena. If $v=c$, then $-\infty+\infty=2 n \pi \boldsymbol{i}$. This seems to be something like a quantum renormalization of infinities that might occur when the space is born, as already suggested in the section of the redshift.

Expressions (25) (28) might suggest a hypothesis that the superposition (simultaneous existence of different states), the wave function collapse (instant collapse into a single state), the entanglement (nonlocal correlation at a distance), the uncertainty (position and momentum cannot be known simultaneously), or the delayed choice experiment (the present affects the past) is related, through the imaginary unit, to the phenomenon of the infinite velocity, though this breaks the special relativity. However, the breakdown of the Lorentz invariant is not allowed to appear in the real world (an interview with John Bell) or might be interpreted as a mathematical phenomenon (an interview with Alain Aspect), as reported by Davies and Brown (1986). The phenomenon of the infinite velocity might be trans- 
formed into the quantum probability wave in order to prohibit the breakdown of the special relativity. The quantum wave function might be a field that has a potential for becoming a particle. By measuring, the quantum superposition of several states, that looks mysterious when not observed, collapses into a single state under the corresponding probability of being found there. The reality of quantum phenomena might be due to the spreading over real and imaginary numbers, a complex number essential to wave phenomena.

\section{CONCLUSIONS}

Mathematical structures common to the animal growth analysis and the space expansion analysis are as follows; the existence of the expression composed of the function and its first and second derivatives, and the existence of the inflection point.

\section{REFERENCES}

Albrecht, A. and P. J. Steinhardt 1982 Cosmology for grand unified theories with radiatively induced symmetry breaking. Phys. Rev. Lett., 48: 1220-1223

Barrow, J. D. 2011 The Book of Universes (The Bodley Head, London). Japanese Translation by H. Hayashi and M. Hayashi for publication (2013), Seidosha Inc., Tokyo

Bedran, M. L. 2002 A comparison between the Doppler and cosmological redshifts. Am. J. Phys., 70: 406-408

Bertalanffy, L. von. 1949 Problems of organic growth. Nature, 163: $156-158$

Bertalanffy, L. von. 1957 Quantitative laws in metabolism and growth. Quart. Rev. Biol., 32: 217-231

Bondi, H. 1964 Relativity and Common Sense (Doubleday and Company Inc., USA). Japanese Translation by T. Yamanouchi for publication (1967), Kawade Shobo Publishers, Tokyo

Brody, S. 1945 Time relations of growth of individuals and populations. In "Bioenergetics and Growth - with special reference to the efficiency complex in domestic animals", Reinhold Publishing Corporation, New York, pp. 484-574

Bunn E. F. and D. W. Hogg 2009 The kinematic origin of the cosmological redshift. arXiv:0808.1081v2

Chodorowski, M. J. 2006 Is space really expanding? A counterexample. arXiv.astro-ph/0601171v2

Davies, P. C. W. and J. R. Brown 1986 The Ghost in the Atom. Cambridge University Press, Cambridge.

Davis, T. M. 2010a Cosmological confusion. Oxford RAL, 9 June 2010.

Davis, T. M. 2010b Is the universe leaking energy? Sci. Am., 7: 33-39

Davis, T. M. and C. H. Lineweaver 2004 Expanding confusion: common misconceptions of cosmological horizons and the superluminal expansion of the universe. Pub. Astron. Soc. Aust., 21: 97-109

Guth, A. H. 1981 Inflationary universe: a possible solution to the horizon and flatness problems. Phys. Rev. D, 23: 347-356

Kazanas, D. 1980 Dynamics of the universe and spontaneous symmetry breaking. Astrophys. J., 241: L59-L63

Li, M. 2014 Understanding the gravitational and cosmological redshifts as Doppler shifts by gravitational phase factors. arXiv:
$1401.5337 \mathrm{v} 2$

Linde, A. D. 1982 A new inflationary universe scenario: a possible solution of the horizon, flatness, homogeneity, isotropy and primordial monopole problems. Phys. Lett., 108B: 389-393

Lineweaver, C. H. and T. M. Davis 2005 Misconceptions about the big bang. Sci. Am., 3: 36-45

May, X. and P. Yu 2013 Cosmology should directly use the Doppler's formula to calculate the red shift of Ia supernova. Int. J. Astron. Astrophys., 3: 303-317

Osumi, S. and Y. Ishikawa 1983 Applicability of the Richards growth function to analysis of growth of tree. Sci. Rep. Kyoto Pref. Univ. Agric. 35: 49-76 (written in Japanese with English summary)

Perlmutter, S. 2011 Measuring the acceleration of the cosmic expansion using supernovae. Nobel Lecture (December 8, 2011), pp. 3-40

Perlmutter, S. 2015 What we learn when we learn that the universe is accelerating. Lecture at 65th Lindau Nobel Laureate Meeting (June 29, 2015).

Perlmutter, S., G. Aldering, G. Goldhaber, R. A. Knop, P. Nugent, P. G. Castro, S. Deustua, S. Fabbro, A. Goobar, D. E. Groom, I. M. Hook, A. G. Kim, M. Y. Kim, J. C. Lee, N. J. Nunes, R. Pain, C. R. Pennypacker, R. Quimby, C. Lidman, R. S. Ellis, M. Irwin, R. G. McMahon, P. Ruiz-Lapuente, N. Walton, B. Schaefer, B. J. Boyle, A. V. Filippenko, T. Matheson, A. S. Fruchter, N. Panagia, H. J. M. Newberg and W. J. Couch 1999 Measurements of $\Omega$ and $\Lambda$ from 42 high-redshift supernovae. Astrophys. J., 517: 565-586

Pierseaux, Y. 2014 Minkowskian solution of general relativity with cosmological constant and the accelerating universe. J. Mod. Phys., 5: 1725-1732

Richards, F. J. 1959 A flexible growth function for empirical use. J. Exp. Bot. 10: 290-300

Riess, A. G., A. V. Filippenko, P. Challis, A. Clocchiatti, A. Diercks, P. M. Garnavich, R. L. Gilliland, C. J. Hogan, S. Jha, R. P. Kirshner, B. Leibundgut, M. M. Phillips, D. Reiss, B. P. Schmidt, R. A. Schommer, R. C. Smith, J. Spyromilio, C. Stubbs, N. B. Suntzeff and J. Tonry 1998 Observational evidence from supernovae for an accelerating universe and a cosmological constant. Astron. J., 116: 1009-1038

Sato, K. 1981 First-order phase transition of a vacuum and the expansion of the universe. Mon. Not. R. Astron. Soc., 195 467-479

Shimojo, M. 2014 An application of Bondi K-factor to the preliminary investigation into some natural phenomena. J. Fac. Agr., Kyushu Univ., 59: 301-303

Shimojo, M. 2015a A size expansion analysis using a toy model based on the equivalence between Bondi K-factor and exponential function. J. Fac. Agr., Kyushu Univ., 60: 93-96

Shimojo, M. 2015b Problems in size expansion analysis based on hypothetical relationships between scale factor, exponential function and Bondi K-factor. J. Fac. Agr., Kyushu Univ., 60 399-403

Shimojo, M., Y. Nakano, M. Tobisa and T. Shao 2012 Deriving five growth functions from Bertalanffy function based on symmetry and complexity. J. Fac. Agr., Kyushu Univ., 57: 151-152

Starobinsky, A. A. 1980 A new type of isotropic cosmological models without singularity. Phys. Lett., 91B: 99-102

Tegmark, M. 2014 Our Mathematical Universe. Alfred A. Knopf, Inc., New York

Tsujikawa, S. 2013 Lecture on Modern Cosmology. Saiensu-sha Co., Ltd. Publishers (written in Japanese)

Weinberg, S. 2008 Cosmology. Oxford University Press Inc., New York 